\title{
Revisión de modelos de madurez en la gestión de los procesos de negocios
}

\author{
Review of maturity models in business processes management \\ Gabriel Páez ${ }^{1} \quad$ Claudia Rohvein $^{1 *} \quad$ Diana Paravie $^{1} \quad$ Mario Jaureguiberry $^{1}$ \\ Recibido 9 de junio de 2017, aceptado 20 de noviembre de 2017 \\ Received: June 9, 2017 Accepted: November 20, 2017
}

\begin{abstract}
RESUMEN
El presente trabajo tiene como objetivo caracterizar y comparar una serie de modelos de madurez de sistemas de gestión disponibles en la literatura y determinar su factible uso en pymes. Para ello se definen ocho criterios en función a bibliografía de referencia. Ellos son dominio de aplicación, propósito de uso, cantidad de dimensiones, cantidad de niveles, descripción por niveles, procedimiento de aplicación, estilo de representación gráfica y complejidad de uso.

Los resultados demuestran que los modelos de Fisher y la Norma ISO 9004 reflejan ser los más indicados para pymes debido principalmente a su baja complejidad de uso y a su representación en dos dimensiones, en donde además se especifica el nivel de desempeño actual y se muestra la ruta de mejoramiento por medio de un perfil de desempeño lineal.

Como contrapartida no disponen de un procedimiento de aplicación y un instrumento para relevar los datos. No obstante se destaca que sólo CCMI y EFQM disponen de un procedimiento de aplicación pero son más complejos.
\end{abstract}

Palabras clave: Sistemas de gestión, modelo de madurez, pymes.

\begin{abstract}
The aim of present study is characterize and compare a series of maturity models for management systems available in the literature and identify feasible use in SMEs. According to reference bibliography, eight criteria are defined for this purpose. They are the domain of application, purpose of use, number of dimensions, number of levels, description by levels, application procedure, graphic style and complexity of use.

The results show that Fisher models and the standard ISO 9004 reflect be best for SMEs mainly due to its low complexity of use and its representation in two dimensions, where also specifies the current performance level and it is displays the path of improvement by means of a linear performance profile.

In return they do not have a procedure of application and an instrument to relieve data. However, only CCMI and EFQM have a procedure of application but they are more complicated..
\end{abstract}

Keywords: Management systems, maturity model, SMEs.

\footnotetext{
1 Dpto. Ing. Industrial. Universidad Nacional del Centro de la Provincia de Buenos Aires/ Facultad de Ingeniería. Av. del Valle 5737. Olavarría, Provincia de Buenos Aires, Argentina. E-mail: gabrieladrianpaez@gmail.com; crohvein@fio.unicen.edu.ar; dparavie@fio.unicen.edu.ar; segumar@fio.unicen.edu.ar

* Autor de Correspondencia
} 


\section{INTRODUCCIÓN}

Las pequeñas y medianas empresas del sector industrial desempeñan un papel muy importante en la economía, principalmente por su contribución en la generación de empleo y por su aporte al proceso de renovación del tejido productivo. No obstante, según [1] en su Informe Anual 2015 - 2016, la principal dificultad de las pymes argentinas en la actualidad se relaciona con el incremento de sus costos productivos en un ambiente recesivo y la consecuente disminución de su rentabilidad.

La literatura clave sobre el concepto de gestión de procesos empresariales y los modelos relacionados con la calidad sugieren que las organizaciones pueden mejorar su desempeño global adoptando un enfoque de procesos [2].

La atención a los procesos ha aumentado y las organizaciones están trabajando en la implementación de acciones para mejorar sus procesos de negocios. Sin embargo el desarrollo de los mismos no implica que sean excelentes o al menos buenos. La noción de madurez es introducida como una medida para indicar cuán excelente es el desempeño de un proceso de negocio. En general un modelo de madurez es una herramienta para evaluar y mejorar habilidades, capacidades y competencias [3].

La gestión y mejora de los procesos de negocios son una tarea central del diseño organizacional. Por ello los modelos de madurez están recibiendo especial atención y se ve un crecimiento en el interés académico [4].

En [5] se define un modelo de madurez como un modelo conceptual que consiste en una secuencia de niveles de madurez discretos para una clase de procesos en uno o más dominios de negocio y que debe representar un camino evolutivo deseado o esperado.

El comité técnico ISO en 2009 define una organización madura como aquella que tiene un desempeño eficaz y eficiente logrando el éxito sostenido con acciones como comprender y satisfacer las necesidades y expectativas de los clientes, realizar el seguimiento de los cambios en el entorno de la organización, identificar posibles áreas de mejora e innovación, definir e implementar estrategias y políticas, establecer y desplegar objetivos pertinentes, gestionar sus procesos y sus recursos, demostrar confianza en las personas, aumentar la motivación, el compromiso y la participación, y establecer relaciones beneficiosas con las partes interesadas [6].

La madurez se ha estudiado desde el punto de vista de los sistemas de gestión de calidad, programas de excelencia y a partir del concepto de Bussiness Process Managment (BPM) [7]. En [8] se define BPM como todos los esfuerzos de una organización para analizar y mejorar continuamente las actividades fundamentales, tales como la fabricación, comercialización, comunicaciones y otros elementos importantes de las operaciones de la empresa.

En [9] se señala que al realizar programas de mejoras las organizaciones se percatan que deben hacer muchos cambios en los procesos, pero se encuentran inseguras respecto a qué cambiar exactamente, en qué medida y cuándo. Los modelos de madurez deben estar formulados de manera que puedan mostrar por donde comenzar, asegurar el progreso y anticipar los resultados.

En [10] se estudió el grado de avance de la bibliografía sobre los modelos de madurez. Para ello basó su búsqueda entre los años 1990 y 2014 para identificar los estudios centrándose en su desarrollo, validación y aplicación. Concluyen que el estado actual de la investigación sobre la madurez de las organizaciones está en sus primeras fases, y la literatura académica carece de aplicaciones metódicas de muchos modelos que se han propuesto.

Dado el interés por parte de las organizaciones en el uso de modelos que ayuden a encaminar el desempeño de los procesos de negocios y su recomendada efectividad, el objetivo del presente trabajo es caracterizar y comparar una serie de modelos de madurez de sistemas de gestión disponibles en la literatura y determinar su factible uso en pymes.

\section{METODOLOGÍA}

Con el propósito de llevar a cabo una revisión bibliográfica adecuada de los modelos de madurez de sistemas de gestión existentes, sus características principales y funcionalidades, se desarrollan las siguientes etapas.

1. Establecimiento de la pregunta a responder por medio de la investigación.

2. Búsqueda de la información.

3. Selección delos modelos aincluirenla comparación. 
4. Definición de los criterios para la caracterización.

5. Construcción de la caracterización y comparación de los modelos seleccionados.

6. Desarrollo de conclusiones a partir de la comparación de modelos.

Primeramente se define el interrogante con la siguiente expresión: ¿cuáles modelos de madurez de sistemas de gestión existentes son adecuados para ser utilizados por empresas pymes industriales?

Para realizar la búsqueda de información se consultó la base de datos Google Académico, dentro de la cual se contemplaron sólo los documentos correspondientes a páginas en inglés y español en el período 2000 a 2016 como criterio de búsqueda. Las palabras clave utilizadas para la construcción de las ecuaciones de búsqueda fueron maturity models, process maturity, BPM maturity's measurements. Se utilizaron combinaciones de estas palabras en inglés y español y se usó el operador lógico OR. Adicionalmente se hizo una revisión de los documentos citados en cada modelo, lo cual permitió identificar autores reconocidos en el desarrollo histórico y conceptual de estos modelos.

Al momento de la selección de los modelos, se decidió incluir para el análisis estudios que desarrollan el concepto de calidad total como, el EFQM (Fundación Europea para la Gestión de la Calidad) e ISO 9004 por ser modelos muy difundidos en la búsqueda de la mejora bajo el enfoque de procesos.

La Tabla 1 muestra los modelos seleccionados.

Tabla 1. Modelos de madurez estudiados.

\begin{tabular}{|c|c|c|}
\hline Modelo de madurez & Año & Autor \\
\hline $\begin{array}{l}\text { Integración de modelos de } \\
\text { madurez de capacidades } \\
(\mathrm{CMMI})\end{array}$ & 2000 & $\begin{array}{l}\text { SEI: Software } \\
\text { Engineering } \\
\text { Institute }\end{array}$ \\
\hline Norma ISO 9004 & 2009 & ISO \\
\hline $\begin{array}{l}\text { Modelo EFQM de } \\
\text { excelencia (EFQM) }\end{array}$ & 1991 & $\begin{array}{l}\text { Fundación Europea } \\
\text { para la Gestión de la } \\
\text { Calidad }\end{array}$ \\
\hline $\begin{array}{l}\text { Modelo de madurez } \\
\text { de procesos y empresa } \\
\text { (PEMM) }\end{array}$ & 2006-2007 & Michael Hammer \\
\hline $\begin{array}{l}\text { Modelo de madurez de } \\
\text { procesos de negocio } \\
\text { (BPMM) }\end{array}$ & 2004 & David Fisher \\
\hline $\begin{array}{l}\text { Modelo de madurez de } \\
\text { procesos de negocio } \\
\text { (BPMM-OMG) }\end{array}$ & 2008 & $\begin{array}{l}\text { Object managment } \\
\text { Group (OMG): } \\
\text { Charlie Weber, } \\
\text { Bill Curtis y Tony } \\
\text { Gardiner }\end{array}$ \\
\hline
\end{tabular}

\begin{tabular}{|l|c|l|}
\hline $\begin{array}{l}\text { Modelo de madurez } \\
\text { holístico para procesos de } \\
\text { negocios (BPMM) }\end{array}$ & 2005 & $\begin{array}{l}\text { Tonia De Bruin \& } \\
\text { Michael Rosemann }\end{array}$ \\
\hline $\begin{array}{l}\text { Modelo de madurez } \\
\text { orientado hacia los } \\
\text { procesos de negocios } \\
\text { (BPOMM) }\end{array}$ & 2009 & Kevin McCormack \\
\hline $\begin{array}{l}\text { Evaluación de la madurez } \\
\text { de la gestión de procesos } \\
\text { (PMMA) }\end{array}$ & 2010 & Michael Rohloff \\
\hline $\begin{array}{l}\text { Modelo de madurez de } \\
\text { procesos de negocio } \\
\text { (BPMM) }\end{array}$ & 2007 & $\begin{array}{l}\text { Jihyun Lee, } \\
\text { Danhyung Lee, y } \\
\text { Sungwon Kang }\end{array}$ \\
\hline
\end{tabular}

Fuente: Elaboración propia.

La Tabla 1 muestra que la comparación se centra en modelos que analicen los procesos de negocios y su gestión, razón por la cual aparecen incorporados la Norma ISO 9004 y EFQM, ya que se fundamentan en el enfoque de procesos. Allí se resume los autores que han desarrollado estos modelos en el período de tiempo acotado en la búsqueda.

En cuanto a la definición de los criterios para la comparación de los modelos, se consideraron como base criterios utilizados por varios autores referentes en la temática [3-4, 7, 9, 11-12].

El criterio "Dominio de aplicación" se refiere a si el modelo es genérico o específico. Se considera genérico cuando puede ser utilizado por cualquier tipo de empresa, sea de servicio o manufactura. En cambio específico es cuando sólo se aplica a un sector productivo en particular [7, 13-14]. Según [4] su alcance o dominio de aplicación debe quedar explícito en la documentación del modelo.

El criterio "Propósito de uso" identifica si el propósito para el cual se pretende usar el modelo de madurez es descriptivo o prescriptivo [11-12]. El propósito es descriptivo si se utiliza como herramienta de diagnóstico permitiendo que los niveles de madurez asignados puedan ser reportados a las partes interesadas internas y externas [15]. De otro modo, un modelo de madurez sirve a un propósito prescriptivo de uso si indica cómo identificar los niveles de madurez deseables y proporciona directrices sobre medidas de mejora [5].

El criterio "Cantidad de dimensiones" se refiere a la complejidad del modelo en su cantidad de ejes, estableciendo la cuadrícula o el esquema general donde se enmarcarán los detalles del modelo [7].

El criterio "Cantidad de niveles" atiende el número de etapas que el modelo considera se deben recorrer 
desde una etapa de madurez básica hasta alcanzar un estado de superioridad.

El criterio "Descripción por niveles" define si el modelo proporciona una descripción detallada en los distintos niveles de madurez para cada uno de las dimensiones abordadas. En [4] se menciona que las descripciones en los niveles son un buen indicador para determinar si los modelos abordan la condición de las prácticas BPM, la condición de los procesos, o ambos.

El criterio "Procedimiento de aplicación" consulta si existen instructivos de aplicación y si se encuentran disponibles. Los mismos facilitan la implementación de los modelos de madurez [9]. Este procedimiento debe proporcionar información sobre cómo adaptarse según las características situacionales específicas de cada organización [4].

El criterio "Estilo de representación gráfica" hace referencia a la forma en que se presenta el nivel de desempeño actual y cómo se interpreta la ruta de mejoramiento para la organización [7].

El criterio "Complejidad de uso" considera la dificultad de aplicación del modelo de madurez.

El criterio "Aplicable a pymes" responde al interrogante: ¿el modelo de madurez en análisis puede ser utilizado en empresas pymes?. Para esto se valora primordialmente que la complejidad de uso sea baja-media o caso contrario disponga un procedimiento de aplicación. Esto se acompaña con un estilo de representación gráfica amigable en donde se pueda visualizar el desempeño actual y la ruta de mejoramiento futura.

Definidos los criterios de comparación, se presenta una breve descripción de los modelos de madurez seleccionados para luego desarrollar una tabla comparativa de mismos que se muestra al final del apartado Resultados.

De los resultados de dicha tabla se efectúa el análisis y las conclusiones sobre la caracterización y comparación realizada, definiéndose los modelos más adecuados para su aplicación en el contexto empresarial pyme.

\section{RESULTADOS}

\section{Caracterización de modelos}

A continuación, se describen los modelos de madurez seleccionados siguiendo el orden de la Tabla 1.

\section{Integración de modelos de madurez de capacidades (CMMI) [16]}

El modelo de integración de madurez de capacidades o Capability Maturity Model Integration (CMMI) [16], diseñado por Software Engineering Institute, es el estándar de calidad más utilizado a nivel internacional por las organizaciones desarrolladoras de software, aunque su uso no se limita solamente a este tipo de organización. Este modelo es utilizado como base para el desarrollo de muchos de los modelos de madurez que se encuentran disponibles actualmente, [16-20]. Su propósito es ayudar a las organizaciones a mejorar sus procesos de desarrollo y de mantenimiento, tanto para los productos como para los servicios.

CMMI utiliza para su evaluación un grupo de áreas de proceso que contienen objetivos y prácticas definidas. De su cumplimiento dependerá el nivel de madurez o capacidad que se asigne. En conjunto con el modelo de madurez, se desarrolló un método para la evaluación, denominado Standard CMMI Appraisal Method for Process Improvement (SCAMPI).

Una característica distintiva del modelo es que permite dos caminos para la mejora y evaluación de procesos, llamados representación continua y escalonada.

La primera caracteriza la mejora de la organización relativa a un área de proceso individual, mediante niveles de capacidad. La segunda caracteriza la mejora de la organización respecto a un conjunto de áreas de proceso por medio de niveles de capacidad.

La Tabla 2 muestra las definiciones de cada nivel de capacidad o madurez de las representaciones continua y escalonada.

Tabla 2. Niveles de capacidad y madurez de CCMI.

\begin{tabular}{|l|c|c|}
\hline Nivel & Niveles de capacidad & Niveles de madurez \\
\hline 0 & Incompleto & No tiene \\
\hline 1 & Ejecutado & Inicial \\
\hline 2 & Administrado & Administrado \\
\hline 3 & Definido & Definido \\
\hline 4 & $\begin{array}{c}\text { Administrado } \\
\text { cuantitativamente }\end{array}$ & $\begin{array}{c}\text { Administrado } \\
\text { cuantitativamente }\end{array}$ \\
\hline 5 & En optimización & En optimización \\
\hline
\end{tabular}

Fuente: [16]. 
En [7] se menciona como principales fortalezas del CMMI que éste asegura que los procesos sean repetibles por las prácticas de institucionalización y exige una transición del 'aprendizaje individual' al 'aprendizaje de la organización'. Igualmente, es una guía detallada para la mejora a través de los niveles de madurez (escalonada) y capacidad (continua) que permite priorizar los esfuerzos iniciales.

Como aspectos negativos, se pueden mencionar que es un modelo excesivamente detallado para algunas organizaciones, difícil de entender y requiere una alta inversión para ser completamente implementado. En [21], se critica al modelo por ignorar la importancia de las personas involucradas en el proceso, asumiendo que los procesos pueden de alguna manera hacer que la excelencia individual sea menos importante.

\section{Norma ISO 9004 [6]}

El comité técnico de ISO define la Norma ISO 9004:2009 bajo el título: Gestión para el éxito sostenido de una organización - Enfoque de gestión de la calidad. Esta Norma Internacional promueve la autoevaluación como una herramienta importante para la revisión del nivel de madurez de la organización, abarcando su liderazgo, estrategia, sistema de gestión, recursos y procesos, para identificar áreas de fortalezas, debilidades y oportunidades tanto para la mejora, como para la innovación.

Del modelo sobresalen dos elementos principales, la orientación a la gestión y la autoevaluación. Esta orientación proporciona un enfoque más amplio sobre la gestión de la calidad que la Norma ISO 9001, trata las necesidades y las expectativas de todas las partes interesadas pertinentes y proporciona orientación para la mejora sistemática y continua del desempeño global de la organización.

La autoevaluación es una revisión exhaustiva y sistemática de las actividades de la organización y de su desempeño en relación con su grado de madurez. Está preparada para proveer un enfoque simple y fácil de usar. Se debería utilizar para determinar las fortalezas y debilidades de la organización en términos de su desempeño, así como de sus mejores prácticas, tanto a nivel general como a nivel de sus procesos individuales.

Esta herramienta de autoevaluación utiliza cinco niveles de madurez. En el anexo de ISO 9004: 2009 se disponen las tablas de autoevaluación.
La implementación de la Norma trae beneficios a la gestión porque cubre todos los procesos de la empresa, permitiendo integrar los elementos adicionales de gestión como medio ambiente, seguridad y salud ocupacional.

Las ventajas de implementar las Normas ISO 9004 son reconocimiento internacional y amplia aplicabilidad en cualquier industria y entorno. Por otro lado, las desventajas son que hay pocas directrices para su implementación en algunas industrias o campos específicos a causa de la amplia aplicabilidad del estándar ISO, tampoco existen directrices para su aplicación en una división o en una sucursal de una gran empresa, se limita en entregar un mapa para implementar el proceso de mejora y no se sabe qué áreas abordar primero y cuáles después.

\section{Modelo EFQM de excelencia (EFQM) [22]}

El Modelo EFQM de excelencia fue creado en 1991 por la European Foundation for Quality Management. El mismo además de brindar orientación para la gestión de excelencia, puede utilizarse para realizar una autoevaluación, una evaluación por parte de un tercero, para actividades de benchmarking como la base para presentarse al Premio Europeo a la Calidad.

La Fundación Europea para la Gestión de Calidad define al modelo EFQM como un marco de trabajo descriptivo basado en conceptos fundamentales de excelencia, criterios fundamentales de excelencia y un esquema lógico REDER.

Los conceptos fundamentales constituyen la base del modelo y son principios básicos para describir las características de una cultura de gestión excelente: orientación hacia los resultados, orientación hacia el cliente, liderazgo y coherencia, gestión por procesos y hechos, desarrollo e implicación de las personas, proceso continuo de aprendizaje, innovación y mejora, desarrollo de alianzas y responsabilidad social de la organización.

Los criterios fundamentales son nueve y pueden utilizarse para evaluar el progreso de una organización hacia la excelencia. Se fundamenta en la premisa según la cual: los Resultados excelentes en el rendimiento general de una organización, en sus clientes, personas y en la sociedad en la que actúa, se logran mediante un Liderazgo que dirija e impulse la Política y estrategia, que se hará realidad a través de las Personas, las Alianzas y recursos, y los Procesos. 
Para desarrollar los criterios en detalle, cada uno va acompañado de un número variable de subcriterios que tienen que considerarse a la hora de realizar una evaluación. Cada subcriterio lleva consigo una lista de áreas a abordar, que no es exhaustiva ni obligatoria, sino prácticas de referencia de gestión excelente. Agrupados en dos categorías, agentes facilitadores y resultados [22].

La matriz de puntuación REDER es el método de evaluación utilizado para puntuar los documentos de solicitud que se presentan al Premio Europeo a la Calidad y debe abordarse para evaluar los criterios clave, es decir los agentes facilitadores y los resultados.

Una vez evaluadas todas las variables del esquema REDER se está en disposición de asignar la puntuación correspondiente a cada criterio y subcriterio de acuerdo con una escala que va de 0 a 100 y que reparte los 1000 puntos máximos a los que puede optar una organización.

La autoevaluación permite realizar un diagnóstico y establecer en qué nivel se encuentra la organización, en su camino hacia la excelencia, e identificar, analizar y priorizar las oportunidades que en ese momento se le presentan para continuar avanzando.

En [3] destaca que esquema lógico REDER proporciona un método simplista, pero eficaz, de comunicar los datos de autoevaluación a la alta dirección para fines de evaluación comparativa. Por otro lado, critica que el modelo no tiene las etapas de maduración descriptas, lo que no permite una buena representación del resultado obtenido.

\section{Modelo de madurez de proceso y de empresa (PEMM) de Hammer [23]}

El modelo de madurez de proceso y de empresa o MMPE, también conocido como PEMM por sus siglas en inglés (Performance Engineering Maturity Model) propuesto por David Hammer (2007), es utilizado para evaluar los niveles de la ejecución y procesos de la organización tanto a organizaciones como a procesos de desarrollo tecnológicos.

Según [23], las empresas necesitan estar seguras que sus procesos de negocio se vuelven más maduros, en otras palabras, que entreguen un alto desempeño que se mantenga en el tiempo. Para lograrlo, deben desarrollar dos tipos de elementos: facilitadores de proceso, que operan en los procesos individuales, y capacidades de empresa, que se aplican a organizaciones completas. El modelo describe cinco facilitadores de proceso: diseño, ejecutores, responsable, infraestructura e indicadores

En [23] se explica que los facilitadores son mutuamente dependientes, si alguno falta, los demás resultarán ineficaces.

Por otro lado, para desarrollar procesos de alto desempeño, las empresas deben ofrecer ambientes que respalden las iniciativas. Para esto, deben poseer o desarrollar capacidades organizacionales en estas áreas: liderazgo, cultura, experticia y gobernabilidad.

Capacidades organizacionales más fuertes resultan en facilitadores más fuertes. Las empresas pueden utilizar conjuntamente los facilitadores y las capacidades para planificar y evaluar el progreso de las transformaciones basadas en procesos. En [23] se sugiere priorizar aquellas áreas de los facilitadores de procesos o de las capacidades de la empresa que representan obstáculos al desempeño del proceso.

Para los facilitadores y para las capacidades se definen cuatro niveles graduales de fortaleza. Los ejecutivos pueden evaluar los niveles de madurez de las capacidades de la empresa (E-1, E-2, E-3, E-4) y los niveles de madurez de los facilitadores de procesos (P-1, P-2, P-3, P-4) estimando si cada proposición del modelo es en gran medida cierta, algo cierta o en gran medida no cierta respecto a sus organizaciones.

En [9] se menciona los siguientes aspectos positivos del modelo: se aplica a empresas de cualquier sector; identifica las características que deben tener todo proceso y toda empresa; se puede aplicar a todos los procesos de la empresa; es fácil de utilizar y la simplicidad del modelo permite a las personas aplicarlo por sí mismas.

En [17] se critica que si bien PEMM describe cómo varios aspectos de una empresa caracterizan diferentes etapas de la madurez del proceso, no proporciona una hoja de ruta de mejora de las prácticas para avanzar a niveles más altos. En consecuencia, el uso de tales modelos es principalmente de diagnóstico. Por otro lado, se reconoce que, como todos los modelos simples, es demasiado general por lo que aumenta la subjetividad al evaluar.

Además, al realizar un análisis de las descripciones del nivel 1 de madurez para los facilitadores de proceso y 
las capacidades de la empresa se encuentra que éstas son muy exigentes para pequeñas empresas.

Modelo de madurez de procesos de negocio (BPMM) de Fisher [24]

El modelo de madurez de proceso de negocio de David Fisher se presenta como una herramienta capaz de proporcionar un balance entre una representación simple que cualquier persona pueda entender fácilmente y un modelo que contenga el suficiente grado de detalle como para proveer ideas que conduzcan a acciones específicas a utilizar por las organizaciones que deseen mejorar su desempeño.

El modelo se centra en el análisis de dos dimensiones. La primera dimensión se descompone en cinco componentes que representan el núcleo de la mayoría de las organizaciones. A estas se las denomina "cinco palancas de cambio", y se designan como estrategia, procesos, tecnología, control y personas. La segunda dimensión son los estados de madurez de estas palancas de cambio.

En el modelo, la dimensión "Cinco palancas de cambio" proporciona los componentes sobre los cuales se puede evaluar las capacidades de una organización en particular. A medida que las capacidades avanzan, la compañía puede progresar a través de la segunda dimensión del modelo, esto es, los estados de madurez. Estos estados son: Aislada, Tácticamente integrada, Proceso conducido, Empresa optimizada, Red inteligente de operaciones.

Debe señalarse que las palancas de cambio son mutuamente dependientes, es decir, para poder disfrutar de los beneficios de un estado de madurez mayor, todas las palancas de cambio deben avanzar conjuntamente. Esto proporciona una clara dirección para una empresa en cuanto a lo que necesita mejorar prioritariamente con el fin de alcanzar el estado deseado.

El camino para pasar de un estado al siguiente no es lineal. De hecho, los obstáculos que deben ser superados varían considerablemente de una etapa a la siguiente y mientras cada palanca cambia sustancialmente de estado a estado, diferentes palancas juegan un papel más importante en cada uno de estos pasos progresivos.

El modelo de Fisher tiene como objetivo ayudar a las empresas a identificar sus propias falencias en cada una de las cinco palancas de cambio y como consecuencia identificar acciones específicas para superar esas limitaciones actuales. Finalmente, las empresas alcanzarán los beneficios que se espera de ellas al superar sus limitaciones actuales.

Se pueden mencionar los siguientes aspectos positivos del modelo: estructura simple y fácil de comprender y utilizar; modelo no lineal y multidimensional y las palancas de cambio mutuamente dependientes proporcionan dirección sobre los aspectos a mejorar prioritariamente para lograr una madurez organizacional superior. Se destaca que el modelo considera la integración externa en su último nivel de madurez, rasgo que lo distingue de otros modelos.

Por otra parte, [9] hace las siguientes críticas al modelo: no es muy específico en cuanto a los aspectos a evaluar debido a su simplicidad, lo que aumenta la subjetividad; no se encuentran disponibles procedimientos para su aplicación y la matriz descriptiva del modelo de madurez constituye un punto de partida, pero no es suficiente para evaluación.

\section{Modelo de madurez de proceso de negocios BPMM-OMG [25]}

El modelo BPMM-OMG fue creado por Charlie Weber, Bill Curtis y Tony Gardiner para la organización sin fines de lucro Object Managment Group.

En el BPMM-OMG la evaluación queda determinada a partir de un análisis de declaraciones de prácticas sobre las áreas de proceso, al igual que en modelo CMMI, las cuales pueden ser verdaderas o falsas.

El modelo presenta cinco niveles de madurez: inicial, gestionado, estandarizado, predecible e innovado u optimizado. Cada nivel incluye objetivos, prácticas y sub prácticas que deben implementarse obligatoriamente si se quiere pasar al siguiente nivel.

Las diferentes maneras en que puede ser utilizado este modelo son: como guía de programas de mejoramiento de los procesos de negocio, como evaluación del riesgo con respecto al desarrollo y la implementación de aplicaciones, como evaluación de la capacidad de proveedores y para realizar una evaluación comparativa (benchmarking).

En [10] se destaca que éste es el único modelo que tiene un alto nivel de propiedades prescriptivas y que puede tomarse como la base de referencia de los 
niveles de madurez, cada uno con áreas específicas de proceso, metas, mejores prácticas y medidas de logros para mejoras de madurez. Como contrapartida a esto, debido principalmente al amplio alcance y al enfoque integral, el esfuerzo requerido para adoptar este modelo específicamente para propósitos descriptivos es considerable, lo que también plantea riesgos y dificultades en su adopción en la práctica. En [17] se menciona que el importante papel del soporte de TI no está cubierto en el BPMM [-OMG].

\section{Modelo de madurez holístico para procesos de} negocios (BPMM) de De Bruin y Rosemann [26]

De Bruin y Rosemann realizaron un estudio utilizando la metodología Delphi con organizaciones internacionales expertas en la gestión de procesos de negocios (BPM) a partir del cual construyeron los elementos centrales de su modelo, al que denominaron BPMM: Modelo de madurez holístico para BPM. El modelo busca ampliar y actualizar los modelos de madurez anteriores al abordar los requisitos y las complejidades identificadas en los BPM de manera holística.

En este modelo, la madurez se define a partir de cuatro dimensiones: factores críticos, alcance, cobertura y competencia

Los factores críticos para la evaluación son: información, cultura, responsabilidad, metodología y desempeño.

La dimensión alcance contempla tanto el momento en que se aplica el modelo como la medida en que las prácticas de BPM se extienden a través de la entidad evaluada.

Por último, la madurez se define como una combinación de las dimensiones cobertura y competencia. La dimensión cobertura se refiere a la capacidad dentro de la organización y el grado en que los principios BPM se implementan y practican, mientras que la competencia mide la calidad y la eficacia de BPM en la organización. En otras palabras, la cobertura pregunta hasta qué punto las actividades de BPM de la organización se extienden y la competencia pregunta qué tan bien se llevan a cabo las actividades de BPM en las dimensiones anteriores. Los niveles de madurez se denominan inicial, definido, repetible, gestionado y sostenido.

Como herramienta de diagnóstico, el modelo presenta encuestas con preguntas cuantificadas en escala de cinco puntos, mostrando los resultados en un gráfico de radar. Como aspectos positivos se destaca que proporciona un marco para la evaluación detallada de las capacidades y logros de BPM. Este modelo BPMM se ha aplicado en dos estudios de caso, lo que confirmó su comprensibilidad, relevancia y aplicabilidad.

En [4] se menciona que, al igual que muchos de los modelos de madurez publicados en artículos de investigación, no está suficientemente detallados los criterios de evaluación y las directrices, y es limitado en su potencial para tratar posibles adaptaciones del modelo de madurez en la práctica.

\section{Modelo de madurez orientado hacia los procesos de negocios (BPOMM) de McCormack [27]}

BPO Maturity Model (BPOMM) es un modelo de madurez para BPM desarrollado por K. McCormack en 2007 y actualizado en 2009 [27], [28]. Según el modelo de madurez de McCormack, hay varios componentes o dimensiones de madurez. Los componentes básicos del modelo son la vista de proceso (documentación de los pasos del proceso, las actividades y las tareas), los trabajos de proceso (responsabilidad horizontal y participación en todo el proceso), la gestión y medición de procesos (mediciones de resultados y mejora de procesos).

Además de los componentes básicos, hay dos componentes de soporte. Éstos son la estructura y la cultura, que permiten que los componentes básicos operen interactivamente.

La construcción de BPM de McCormack describe una ruta de cuatro pasos para avanzar sistemáticamente en los procesos de negocio a lo largo de los niveles de madurez llamados ad hoc, definido, vinculado e integrado. Cada paso se basa en el trabajo de los pasos anteriores para aplicar estrategias de mejora que son apropiadas para el nivel de madurez actual [27-28].

En el modelo, se proporciona una versión alternativa de su modelo de madurez que se adapta específicamente para la gestión de la cadena de suministro.

Por otro lado, la guía de evaluación para determinar las puntuaciones de madurez no está disponible, ni tampoco un procedimiento para su implementación. 
Evaluación de la madurez de la gestión de procesos (PMMA) de Rohloff [17]

El modelo llamado "Evaluación de la Madurez de la Gestión de Procesos" (PMMA) [17] fue diseñado por Michael Rohloff, dentro del marco de un proyecto corporativo de la Compañía Siemens, llamado Siemens AG. El PMMA sigue la estructura del modelo CCMI, pero proporciona una evaluación integral de todas las áreas relevantes para BPM basado en un conjunto de nueve criterios o categorías. Como indicador para la madurez del proceso, consideran cinco niveles similares a los del modelo CMMI.

El principal objetivo del PMMA es la identificación de la necesidad de acción y derivación de medidas para el mejoramiento de la gestión de procesos, así como la identificación de los requisitos para el apoyo adicional.

En [4] se destaca que el modelo PMMA se diferencia de otros modelos en que requiere poca documentación.

Como aspecto negativo se puede mencionar que el modelo no tiene disponible procedimiento de aplicación y es difícil de entender, por lo que requiere de expertos para ser aplicado.

\section{Modelo de madurez de proceso de negocio} (BPMM) de Lee et al. [18]

The Business Process Maturity Model [18] (BPMM-Lee) propuesto por Jihyun Lee, Danhyung Lee, and Sungwon Kang es un modelo de madurez de BPM alineado con los términos, niveles de madurez y algunos elementos de Áreas de Proceso Clave (KPAs) del estándar CMMI. Su diferencia con CCMI radica en que este modelo se basa en el principio de que cualquier proceso de negocio consiste esencialmente en actividades pertenecientes a cuatro categorías: Entrada,
Mecanismo, Control y Salida (IMCO por sus siglas en inglés) y por lo tanto se estructuran las Áreas de proceso de manera diferente a CCMI en los distintos niveles de madurez. Estas cuatro categorías se describen en cinco niveles de madurez.

Como parte de la investigación utilizada para la construcción del modelo, se incorporaron los resultados de las investigaciones existentes sobre modelos de madurez y por otro lado se llevó a cabo una encuesta sobre un grupo de empresas que buscan activamente la gestión de procesos empresariales. Como consecuencia, el BPMM-Lee presenta como ventajas combinar características de prácticas del sector empresarial con una estructura en cumplimiento con CMMI. Fue diseñado de tal manera que podría superar las limitaciones de los modelos de madurez orientados al software existentes, reflejando la voz del mercado y las buenas prácticas de empresas que trabajan en la gestión por procesos.

Por otro lado, el modelo tiene las debilidades propias de un modelo surgido de una investigación, no cuenta con un procedimiento de aplicación ni metodología o directrices de evaluación detallados lo que dificulta su adopción en la práctica [17].

\section{Comparación de modelos}

El resultado de la comparación de los modelos de madurez según los criterios citados en la metodología se muestra en la Tabla 3.

Los modelos de madurez CCMI y OMG persiguen un propósito prescriptivo de uso, es decir que indican cómo identificar los niveles de madurez deseables y proporcionan directrices sobre medidas de mejora. El resto de los modelos analizados se utilizan como herramienta de diagnóstico con un propósito de uso descriptivo. 


\begin{tabular}{|c|c|c|c|c|c|c|c|c|c|}
\hline 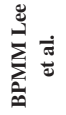 & 递 & 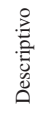 & - & in & $\dot{n}$ & 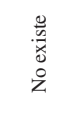 & 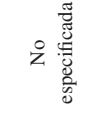 & 嵒 & $\check{z}$ \\
\hline 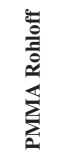 & : & 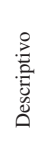 & $\sim$ & in & $\dot{\omega}$ & 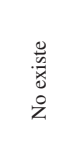 & 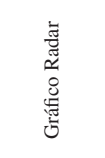 & $\stackrel{\Xi}{4}$ & $\stackrel{\circ}{z}$ \\
\hline 惫 & : & 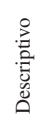 & + & $\checkmark$ & $\bar{n}$ & 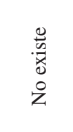 & そ̊ 莺 & $\stackrel{g}{4}$ & $\stackrel{\circ}{z}$ \\
\hline 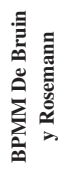 & : & 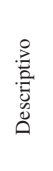 & + & in & $\bar{n}$ & 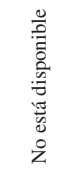 & 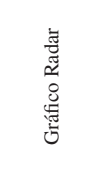 & $\stackrel{\Xi}{4}$ & $\stackrel{\circ}{z}$ \\
\hline $\begin{array}{l}0 \\
\sum_{0}^{0} \\
i \\
\sum_{i=0}^{1}\end{array}$ & 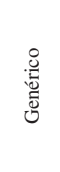 & 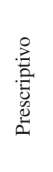 & $\sim$ & in & $\bar{n}$ & 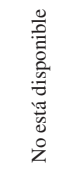 & 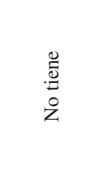 & $\stackrel{\Xi}{4}$ & $\check{z}$ \\
\hline 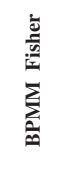 & : & 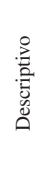 & $\sim$ & in & $\bar{n}$ & 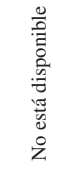 & 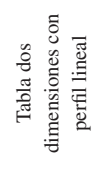 & 迎 & $\dot{i n}$ \\
\hline 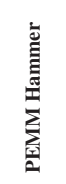 & 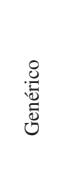 & 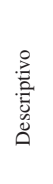 & $m$ & 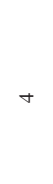 & $\bar{n}$ & 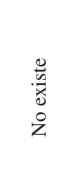 & 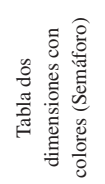 & 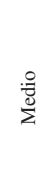 & z \\
\hline $\begin{array}{l}\text { ¿ } \\
\text { 离 }\end{array}$ & 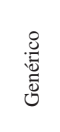 & 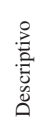 & $\sim$ & 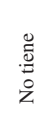 & $\check{z}$ & 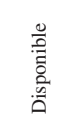 & 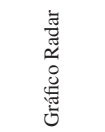 & 胥 & $i n$ \\
\hline $\begin{array}{l}\text { 言 } \\
\text { Oे }\end{array}$ & 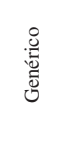 & 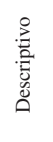 & $\alpha$ & in & $\bar{n}$ & 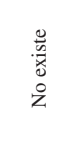 & 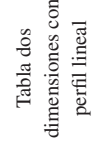 & 邑 & $\vec{n}$ \\
\hline E & 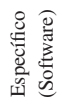 & 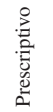 & $N$ & in & $\dot{n}$ & 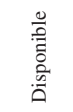 & 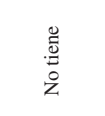 & $\stackrel{\Xi}{4}$ & $i n$ \\
\hline & 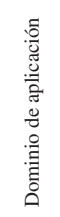 & 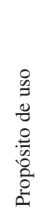 & 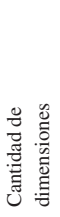 & 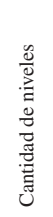 & 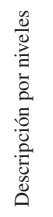 & 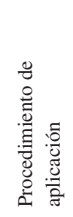 & 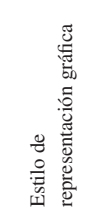 & 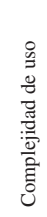 & 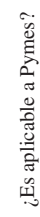 \\
\hline
\end{tabular}


El modelo Lee et al. tiene una sola dimensión por lo que los detalles del mismo se pueden representar en una pirámide con una descripción única para cada nivel.

Hammer desarrolla los detalles de su modelo en un cubo. De Bruin y Roseman y McCormack presentan cuatro dimensiones por lo cual al cubo se adiciona alguna dimensión subdividida.

El resto de los modelos se representan en dos dimensiones donde los detalles se desarrollan en una cuadricula y se encuentran descripciones de cada nivel (primera dimensión) para cada uno de los aspectos a evaluar (segunda dimensión).

El modelo EFQM es el único que no presenta número de niveles ni descripciones en ellos. El número de niveles en todos los modelos varía entre cuatro y cinco.

Sólo los modelos CCMI y EFQM tienen disponible un procedimiento de aplicación.

Los modelos EFQM, De Bruin y Roseman y Rohloff usan el radar como estilo de representación gráfica mientras que la Norma ISO 9004, Fisher y Hammer los hacen por intermedio de la misma tabla de dos dimensiones donde se disponen los detalles del modelo. La Norma ISO 9004 y Fisher muestran la ruta de mejoramiento mediante un perfil de desempeño lineal, de este modo se deben atender primero los sectores que se encuentren con valores de desempeño menor al deseado. Hammer lo hace utilizando colores, relacionados con un semáforo (rojo, amarillo y verde), donde se deben atender prioritariamente los sectores en rojo que son los más críticos.

Del análisis se deriva que los modelos de menor complejidad de uso son Fisher y la Norma ISO 9004, seguidos de EFQM y Hammer. Aunque este último inicia su primer nivel con exigencias de desempeño muy altas.

Finalmente para responder a la pregunta de investigación se visualiza en la Tabla 3 que los modelos CCMI, EFQM, Norma ISO 9004 y Fisher pueden ser utilizados en pymes.

Sin embargo en el caso del CCMI su dominio de aplicación se centra en empresas de software y su complejidad de uso es alta, aunque dispone de un procedimiento de aplicación que puede auxiliar. Por su parte el EFQM tiene una complejidad de uso moderada.

Finalmente el análisis arroja que los modelos de madurez de gestión de mayor factibilidad de uso en pymes son Fisher y la Norma ISO 9004.

\section{CONCLUSIONES}

El trabajo analiza una serie de modelos de madurez de sistemas de gestión, seleccionados mediante una revisión bibliográfica, a través de ocho criterios para determinar la factibilidad de uso en pymes.

Los criterios son dominio de aplicación, propósito de uso, cantidad de dimensiones, cantidad de niveles, descripción por niveles, procedimiento de aplicación, estilo de representación gráfica y complejidad de uso.

Lo modelos de Fisher y la Norma ISO 9004 reflejan ser los más indicados para pymes debido principalmente a que su complejidad de uso es baja. Al representarse en dos dimensiones hace que baje la complejidad del modelo en su cantidad de ejes, estableciendo la cuadricula donde se enmarcarán los detalles del modelo y se encuentran las descripciones de cada nivel para cada uno de los aspectos a evaluar.

Utilizan esta misma cuadricula para especificar el nivel de desempeño actual por medio de un diagnóstico gráfico, mostrando la ruta de mejoramiento mediante un perfil de desempeño lineal. Entendiendo que para interpretar cual debería ser la ruta de mejoramiento para la organización habría que comenzar por los sectores con menor valor de desempeño.

Su dominio de aplicación es genérico y su propósito de uso descriptivo. Si bien su complejidad de uso es baja no se dispone de un procedimiento de aplicación.

Esto hace que al momento de utilizarlo se deba recurrir al armado de algún instrumento que ayude a realizar el diagnóstico y complementar con una metodología de evaluación para valorizar los datos relevados.

Asimismo, al momento de proponer mejoras y definir acciones para transitar la ruta de mejoramiento se debe especificar cómo identificar los niveles de madurez deseables y proporcionar directrices sobre medidas de mejora. 
Se destaca que sólo CCMI y EFQM disponen de un procedimiento de aplicación.

Los resultados arribados permiten hacer uso de los modelos de madurez de gestión para aportar al desempeño de las pymes.

Se anticipa que sería posible aplicarlo en cualquier sector industrial, aunque el interés de los autores sería comenzar con empresas del sector metalmecánico. Teniendo en cuenta que los niveles de madurez de los dos modelos sugeridos son cinco, los parámetros al momento de seleccionar las empresas serían: para el nivel 1 sin certificado de ninguna índole, para el nivel 2 certificado 9001 (como mínimo), para el nivel 3 certificado $9001+14000$ (como mínimo), para el nivel 4 certificado $9001+14000+18000$ (como mínimo) y para el nivel 5 reconocimiento del premio nacional de calidad. Se aceptaría que la empresa esté utilizando el modelo, pero sin lograr los certificados.

La aplicación se realizaría en tres casos que a priori se piensen representen un nivel entre uno y dos, entre dos y tres y entre tres y cuatro.

Por otro lado, la relevancia del presente trabajo se visualiza como paso previo a generar un aporte a los modelos que ayudan a mejorar el desempeño en pymes. Según [29] el complemento entre los sistemas de medición de desempeño (SMD) con los sistemas de gestión facilita su aplicación y genera un aporte al aprendizaje organizacional y la capacidad empresaria. Esta sugerencia da indicios para pensar en realizar un modelo que incorpore características relacionadas con la gestión como un modelo de procesos de negocios (BPM) a los modelos de madurez de SMD disponibles en la bibliografía.

El uso de los SMD permite medir el desempeño organizacional, generando información para la toma de decisiones, cuantificando eficiencia y eficacia. Medir su madurez es útil para guiar su desarrollo tal como se manifiesta en los modelos de Wettstein y Kueng 2002, Van Aken et al. 2005, Tangen 2004 y Aho 2011.

En [30] concluyen que los modelos planteados por Wettstein y Kueng y Aho exponen mayor cantidad de dimensiones y detalles, facilitando la objetividad y aplicación. Asimismo, son recomendables para PyMEs por su aplicación amigable, bajo requerimiento de recursos y tiempo.
Proyectando la aplicación de modelos de madurez de medición de desempeño y de gestión, con aplicabilidad en PyMEs, se debe plantear la futura creación de un modelo integral teórico que combine ambos para medir la alineación entre la madurez de los dos sistemas.

\section{REFERENCIAS}

[1] Fundación Observatorio PyME. "Informe 2015 - 2016. Evolución reciente, situación actual y desafíos para 2017. Tema especial: Indicadores de producción", Fecha de consulta: 15 de mayo de 2017. http://www.observatoriopyme. org.ar/newsite/wp-content/uploads/2016/10/ Informe-FOP-2015-16_Baja.pdf

[2] J. Pérez Fernández de Velasco. "Gestión por procesos". ESIC Editorial. $5^{\circ}$ Edición. Madrid, España. pp. 310. 2012. ISBN: 8473568540 , 9788473568548.

[3] M. Moradi-Moghadam, H. Safari and M. Maleki. "A novel model for business process maturity assessment through combining maturity models with EFQM and ISO 9004:2009”. International Journal Business Process Integration and Management. Vol. 6 $\mathrm{N}^{\circ}$ 2, pp. 167-184. Enero 2013. ISSN online: 1741-8771.

[4] M. Röglinger, J. Pöppelbuß and J. Becker, "Maturity models in business process management". Business Process Management Journal. Vol. 18 No 2, pp. 328-346. 2012. DOI: $10.1108 / 14637151211225225$

[5] J. Becker, R. Knackstedt, y J. Pöppelbuß, "Developing maturity models for IT management", Business \& Information Systems Engineering. Vol. $1 \mathrm{~N}^{\circ} 3$, pp. $213-$ 222. 2009. DOI: $10.1007 / \mathrm{s} 12599-009-0044-5$

[6] ISO 9004. "Gestión para el éxito sostenido de una organización. Enfoque de gestión de la calidad". 2009.

[7] M. D. P. Díaz Jaimes y N. R. Ortiz Pimiento. "Revisión de Modelos de Madurez: Estrategia de Evaluación del Desempeño para Empresas de Manufactura", Revista UIS Ingeniería. Vol. $11 \mathrm{~N}^{\circ} 1$, pp. 55-72. Mayo 2013. ISSN on line: 2145-8456.

[8] P. Trkman. "The critical success factors of business process management". International Journal of Information Management. Vol. 30 $\mathrm{N}^{\mathrm{o}}$ 2, 125-134. 2010. ISSN: 0268-4012. 
[9] E. Pérez-Mergarejo, I. Pérez-Vergara y Y. Rodríguez-Ruíz. "Modelos de madurez y su idoneidad para aplicar en pequeñas y medianas empresas", Ingeniería Industrial. Vol. $35 \mathrm{~N}^{\circ} 2$, pp. 184-198. 2014. ISSN: 0258-5960.

[10] A. Tarhan, O. Turetken y H. A. Reijers. "Business process maturity models: a systematic literature review". Information and Software Technology Journal. Vol. 75, pp. 122-134. 2016. http://dx.doi.org/10.1016/j.infsof.2016.01.010

[11] A. Van Looy, M. De Backer, G. Poels y M. Snoeck. "Choosing the right business process maturity model". Information \& Management. Vol. $50 \mathrm{~N}^{\mathrm{o}}$ 7, pp. 466-488. 2013. https://doi. org/10.1016/j.im.2013.06.002

[12] J. Pöppelbuß and M. Röglinger. "What makes a useful maturity model? a framework of general design principles for maturity models and its demonstration in business process management". European Conference on Information Systems (ECIS). Helsinki, Finland. 2011.

[13] F. Ahlemann, C. Schroeder and F. Teuteberg. "Kompetenz-und Reifegradmodelle für das Projektmanagement". Grundlagen, Vergleich und Einsatz. ISPRI-Arbeitsbericht. $\mathrm{N}^{\circ} 1$ 2005. ISBN: 3-936475-24-5.

[14] M. H. Ofner, K. M. Huener and B. Otto. "Dealing with Complexity: A Method to Adapt and Implement a Maturity Model for Corporate Data Quality Management". Americas Conference on Information Systems (AMCIS). San Francisco, California, USA. 2009.

[15] A.Maier,J.MoultrieandP.J.Clarkson.'Developing maturity grids for assessing organisational capabilities: Practitioner guidance". 4th International Conference on Management Consulting. Vienna, Austria. 2009.

[16] M. B. Chrissis, M. Konrad and S. Srhum. "CMMI. Guía para la integración de procesos y la mejora de productos". Pearson Educación. $2^{\circ}$ Edición. España. pp. 664. 2009. ISBN: 9788478290963

[17] M. Rohloff. "Advances in business process management implementation based on a maturity assessment and best practice exchange". Journal Information Systems and e-Business Management. Vol. $9 \mathrm{~N}^{\circ}$ 3, pp. 383-403. sep. 2011. DOI:10.1007/ s10257-010-0137-1
[18] J. Lee, D. Lee and S. Kang, "An Overview of the Business Process Maturity Model (BPMM)". Advances in Web and Network Technologies, and Information Management. Huang Shan, China. Juanary 2007.

[19] J. Alden and B. Curtis. "Business Process Improvement". Fecha de consulta: 2 de Mayo de 2017. URL: http://www. bptrends.com/publicationfiles/11-06-COLBPM\%26OrganizationalMaturity-CurtisAlden-Final1.pdf

[20] C. Amaya y L. Felipe. "Modelos de capacidad y madurez y la industria del software en Colombia”. Generación. Digital. Vol. 7 N² , pp. 1-4. 2008. ISSN 1909-9223.

[21] M. Von Rosing, H. Von Scheel and A.W. Scheer, "The Complete Business Process Handbook: Body of Knowledge from Process Modeling to BPM". Morgan Kaufmann. Vol. 1, pp. 776. 2014. ISBN: 012800472X, 9780128004722

[22] EFQM. "Introducción a La Excelencia". Fecha de consulta: 4 de mayo de 2017. URL:www. efqm.org

[23] M. Hammer. "La auditoría de proceso", Harvard Business Review. Vol. 85, № 4, pp. 92-104. 2007. ISSN 0717-9952.

[24] D. M. Fisher. "The business process maturity model: a practical approach for identifying opportunities for optimization". Bus. Process Trends. Vol. 9 No 4, pp. 11-15. 2004.

[25] C. Weber, B. Curtis and T. Gardiner, "Business process maturity model (BPMM) version 1.0". Fecha de consulta: 4 de Mayo de 2017. URL:

http://www.omg.org/spec/BPMM/1.0/

[26] T. De Bruin y M. Rosemann. "Towards a business process management maturity model". ECIS 2005 Proceedings of the Thirteenth European Conference on Information Systems. Regensburg, Germany. 26-28 May 2005.

[27] K. McCormack. "Business Process Maturity Theory and Application". BookSurge Publishing. pp. 350. 2007. ISBN: 1-4196-7913-9

[28] K. McCormack, J. Willems, J. van den Bergh, D. Deschoolmeester, P. Willaert, M. Stemberger, R. Skrinjar, P. Trkman, M. Ladeira, M. Valadares de Oliveira, V. Vuksic and N. Vlahovic. "A global investigation of key turning points in business process maturity”. Business Process 
Management Journal. Vol. $15 \mathrm{~N}^{\circ}$ 5, pp. 792815. 2009 DOI: $10.1108 / 14637150910987946$.

[29] U. S. Bititci, P. Garengo, A. Ates and Y S. S. Nudurupati. "Value of maturity models in performance measurement", International Journal of Production Research. Vol. $53 \mathrm{~N}^{\mathrm{o}} 10$, pp. 3062-3085. 2014. DOI: 10.1080/00207543.2014.970709.

[30] M.P. Bertolli, G.Roark, S.Urrutia y F. Chiodi. "Revisión de modelos de madurez en la medición del desempeño" INGE CUC. Vol. $13 \mathrm{~N}^{\circ} 1$, pp. 70-83, 2017. DOI:http://dx.doi.org/10.17981/ ingecuc.13.1.2.017.07 